\title{
Le nettoyage rituel des canaux d'irrigation d'une communauté de la cordillère de Lima (province de Canta, Pérou) : une approche ethnohistorique
}

La limpieza ritual de las acequias de una comunidad de la sierra de Lima (provincia de Canta, Perú): un enfoque etnohistórico

The ritual cleansing of the irrigation canals of a community of the Cordillera of Lima (Province of Canta, Peru): an ethnohistorical approach

\section{Alexandra Carlier}

\section{(2) OpenEdition}

\section{Journals}

Édition électronique

URL : http://journals.openedition.org/bifea/3144

DOI : 10.4000/bifea.3144

ISSN : 2076-5827

Éditeur

Institut Français d'Études Andines

Édition imprimée

Date de publication : 1 août 2008

Pagination : 351-374

ISSN : 0303-7495

Référence électronique

Alexandra Carlier, «Le nettoyage rituel des canaux d'irrigation d'une communauté de la cordillère de Lima (province de Canta, Pérou) : une approche ethnohistorique », Bulletin de l'Institut français d'études andines [En ligne], 37 (2) | 2008, mis en ligne le 01 février 2009, consulté le 29 novembre 2020. URL http://journals.openedition.org/bifea/3144 ; DOl : https://doi.org/10.4000/bifea.3144

\section{(c) (i) $\odot$}

Les contenus du Bulletin de l'Institut français d'études andines sont mis à disposition selon les termes de la licence Creative Commons Attribution - Pas d'Utilisation Commerciale - Pas de Modification 4.0 International. 


\title{
Le nettoyage rituel des canaux d'irrigation d'une communauté de la cordillère de Lima (province de Canta, Pérou) : une approche ethnohistorique
}

\author{
Alexandra Carlier ${ }^{*}$
}

\section{Résumé}

Cet article offre une étude ethnohistorique du nettoyage rituel des canaux d'irrigation de la communauté villageoise de San Pedro de Huacos (province de Canta) située dans les Andes centrales péruviennes. Grâce aux données de terrain récoltées sur la version actuelle et la version ancienne (fin du XIX siècle et début du XXe siècle) de ce rituel et à l'analyse de sources ethnohistoriques provenant de la zone étudiée, nous avons tenté de reconstituer et de comprendre une pratique religieuse locale attestée dès l'époque préhispanique, celle de la vénération des divinités féminines pourvoyeuses d'eau considérées comme gardiennes des canaux d'irrigation.

Mots clés : Andes péruviennes, vallée haute du Chillón, nettoyage rituel des canaux d'irrigation, ethnohistoire, divinités pourvoyeuses d'eau

\section{La limpieza ritual de las acequias de una comunidad de la sierra de Lima (provincia de Canta, Perú): un enfoque etnohistórico}

\section{Resumen}

Este artículo brinda un estudio etnohistórico de la limpieza ritual de las acequias de la comunidad campesina de San Pedro de Huacos (provincia de Canta) ubicada en los Andes Centrales del Perú.

* Doctorante en Histoire de l'Art et Archéologie, Faculté de Philosophie et Lettres, à l'Université Libre de Bruxelles. E-mail : alcarlie@ulb.ac.be 
Con los datos de campo recogidos sobre la versión actual y la versión antigua (finales del siglo XIX y principios del siglo XX) de este ritual y el análisis de fuentes etnohistóricas relativas a la zona estudiada, intentamos reconstituir y comprender una práctica religiosa local atestiguada ya en la época prehispánica, la de la veneración de las divinidades femininas proveedoras de agua consideradas como guardianas de las acequias.

Palabras claves: Andes peruanos, valle alto del Chillón, limpieza ritual de las acequias, etnohistoria, divinidades proveedoras del agua

\title{
The ritual cleansing of the irrigation canals of a community of the Cordillera of Lima (Province of Canta, Peru): an ethnohistorical approach
}

\begin{abstract}
The article presents an ethnohistorical study of the ritual cleansing of the irrigation canals of the community of San Pedro de Huacos (province of Canta) located in the Centrals Peruvian Andes.

Combining the fieldwork data collected on the current version and the old version (late XIXth century and early XXth century) of this ritual with the analysis of ethnohistorical sources relating to the studied zone, we tried to reconstitute and contextualise a local religious practice reported from prehispanic times - that of the veneration of the female water-providing divinities regarded as guardians of the irrigation canals.
\end{abstract}

Key words: The Peruvian Andes, high valley of Chillón, ritual cleansing of the irrigation canals, ethnohistory, water-providing divinities

Pour les populations de la côte et des contreforts andins, l'eau fut toujours une préoccupation majeure. Outre la mise en place de moyens à caractère technique et administratif, les anciens habitants de la côte et de la cordillère avaient recours à des pratiques magicoreligieuses pour s'octroyer les bienfaits de cet élément et pour s'assurer des cultures optimales. Les rituels liés à l'eau d'irrigation et à l'eau de pluie étaient de nature variée et caractérisés par une profonde ambivalence car l'eau était considérée à la fois comme un élément bénéfique et créateur mais aussi comme un élément destructeur lié à la mort.

Nous n'aborderons ici qu'un rituel relatif à l'eau d'irrigation : celui du nettoyage des canaux en milieu montagnard péruvien. La plupart des communautés villageoises de la sierra péruvienne possèdent sur leurs territoires un important réseau de canaux d'irrigation et de réservoirs, datant de l'époque préhispanique, qui leur permettent durant la saison sèche de pallier l'insuffisance en eau de pluie des terres cultivées. Les autorités communautaires organisent au moins une fois par an un travail collectif pour nettoyer les canaux et remédier aux dégâts causés par la pluie et les déplacements du bétail.

Le choix du thème de cet article est fondé sur nos données de terrain récoltées dans la communauté villageoise de San Pedro de Huacos lors de l'étude de ce rituel1,

1 La recherche de terrain, menée dans le cadre d'un mémoire de licence en Histoire de l'Art et Archéologie à I'Université Libre de Bruxelles, s'est déroulée entre août et novembre 2004 sur la rive droite de la vallée haute du Chillón, côte centrale du Pérou, dans deux communautés villageoises voisines : San Pedro de Huacos, 3252 m, et Santiago de Huaros, 3583 m d'altitude (département de Lima, province de Canta, district de Huaros). 
communément appelé limpiacequia ou fiesta al agua (fête de l'eau) dans la vallée haute du Chillón (province de Canta).

Combinant données ethnographiques, archéologiques et historiques2, la recherche avait pour but d'étudier et d'analyser, dans une perspective diachronique comparative, les limpiacequias anciennes et actuelles des populations du haut Chillón. Nous avons privilégié les approches contextualiste et post-processualiste qui nous ont permis d'étudier l'évolution du rituel d'un point de vue formel et fonctionnel, depuis l'époque de l'Intermédiaire récent 3 (1000-1450) jusqu'à nos jours. Notons que nous avons intégré aux reconstitutions du passé des éléments relatifs aux mentalités et au contexte historique.

La recherche a montré qu'il existe dans le rituel actuel de la limpiacequia de la zone montagnarde étudiée des persistances, comme par exemple la vénération de divinités pourvoyeuses d'eau personnifiées par des huacas, qui s'enracinent dans ce que Carrión Cachot (1955) appelle le culte de l'eau dans le Pérou ancien. Les travaux de nombreux américanistes (Carrión Cachot, 1955 ; Duviols, 1977 ; Farfán, 2002 ; Gelles, 2002 ; Llanos \& Osterlings, 1981 ; Mariscotti de Görlitz, 1973 ; Rostworowski, 1978 ; Salomon, 1997 ; Tello \& Miranda, 1923 ; Villar Córdova, 1933) attestent en effet que les populations préhispaniques récentes (1000-1532), et dans ce cas-ci plus particulièrement celles de la cordillère, se sont organisées, à chaque époque, pour s'assurer non seulement la maîtrise de l'eau d'irrigation par la construction d'infrastructures hydrauliques (terrasses de culture, réseaux de canaux d'irrigation, barrages et bassins en maçonnerie de pierre, etc.) et la mise en place de groupes sociopolitiques d'irrigateurs (Sherbondy, 1979 ; 1987) mais aussi son abondance par la vénération de divinités pourvoyeuses d'eau et l'institution de cérémonies en relation avec leur calendrier agricole. Cette affirmation trouve notamment son origine dans des données provenant de sources anciennes, tels que le manuscrit de Huarochirí (Taylor, 1980 : ch. XXXI) ou l'ouvrage du Jésuite Arriaga (1999 [1621] : ch. II). Grâce à ces dernières nous savons que les populations préhispaniques récentes vénéraient des divinités pourvoyeuses d'eau comme maîtresses des eaux, créatrices et gardiennes des canaux et parfois aussi, par analogie, certains animaux qui leur étaient associés.

C'est dans le contexte de cette thèse, suggérant l'existence d'une « relative continuité » du rituel à travers les siècles, que les données présentées ici doivent être comprises. Le présent article s'attachera à étudier la version « ancienne » du rituel (fin du XIXe siècle-début du XX siècle), soit celle qui nous a été commentée et expliquée par nos informateurs privilégiés : les anciens de la communauté de San Pedro de Huacos. Des entretiens, dirigés ou non, ont aussi été menés auprès d'adolescents, d'enfants et de femmes de tous âges afin de constituer un ensemble de variations qui nous a permis d'éviter le piège de la relation individuelle transposée à la sphère collective.

Le rituel sera tout d'abord situé dans une structure calendaire pour comprendre la place qu'il occupe parmi les autres activités agricoles de la communauté. Après une description de

2 D'une part, nous avons décrit et analysé les célébrations actuelles du nettoyage des canaux d'irrigation, datés de I'Intermédiaire récent (1000-1450), de deux communautés villageoises de la vallée haute du Chillón (San Pedro de Huacos et Santiago de Huaros) pour en comparer les données avec celles d'études ethnoarchéologiques et anthropologiques sur le même sujet. D'autre part, nous avons fait une analyse archéologique des établissements de l'Intermédiaire récent et de l'Horizon récent (1450-1532) de la vallée haute du Chillón, complétée par des données historiques et ethnohistoriques (chroniques et manuscrits de l'époque coloniale), en vue de comprendre le cadre sociopolitique dans lequel ces établissements virent se dérouler des cérémonies et des rituels liés à l'eau d'irrigation, à l'instar des communautés villageoises actuelles.

3 Les études archéologiques des sites de la vallée haute du Chillón les datent, pour la plupart, de l'Intermédiaire récent (1000-1450), et précisent que leurs réseaux d'irrigation se situent, généralement, dans les hauteurs (Farfán 2002 ; Silva, 1996 ; Villar Cordóva, 1923, 9-10). 
I'ancienne version de la limpiacequia, telle qu'il nous a été possible de la reconstituer grâce aux entretiens et à notre observation participante du rituel en août 2004, nous en analyserons un aspect particulier : la nature féminine de la divinité locale pourvoyeuse d'eau d'irrigation, appelée la Mamahuaca. Le propos de cette analyse sera de mettre en exergue l'influence des contextes historiques et culturels de la vallée haute du Chillón sur cet aspect du rituel.

Nous sommes bien consciente que les analyses archéologiques, les documents historiques anciens et les enquêtes orales ne donnent accès qu'à certains éléments du contexte religieux des populations de la vallée haute du Chillón. Ils peuvent néanmoins nous mener vers des pistes à suivre afin de comprendre un rituel agricole dont les prémices remontent à la préhistoire locale.

Avant toute chose, nous proposons d'exposer de manière synthétique quelques concepts relatifs à l'eau d'irrigation dans la pensée andine ancienne et actuelle pour comprendre ensuite le rituel annuel de la limpiacequia.

\section{L’EAU DANS LA RÉGION ANDINE}

Dans la pensée andine ancienne, l'eau de pluie ou l'eau d'irrigation était l'apanage de diverses divinités pourvoyeuses d'eau, masculines ou féminines, personnifiées pour la plupart par des huacas. Les populations du Pérou ancien leur rendaient hommage pour recevoir les eaux fertilisantes et bénéfiques aux cultures mais aussi pour se protéger des inondations, des orages et des pluies torrentielles. En effet, les mythes, notamment ceux du manuscrit de Huarochirí (XVII siècle), mentionnent l'existence de divinités masculines pourvoyeuses d'eau d'irrigation. Un thème y est récurent: le chagrin d'une jeune fille causé par le manque d'eau ou les semences desséchées, faute d'irrigation. Cette situation est résolue par le prétendant de la jeune fille, I'Inca ou une divinité principale, qui veut démontrer son pouvoir sur la nature en créant des canaux d'irrigation (Taylor, 1980 : ch. XXXI). Lors des travaux d'irrigation, ces divinités principales sont généralement aidées par des animaux, dont la présence coïncide avec l'apparition des pluies. Il s'agit de crapauds, de serpents, de félins, de renards et aussi d'oiseaux (Carrión Cachot, 1955 : 14).

Dans la religion officielle incaïque du Cuzco, Illapa était considéré comme la divinité dispensatrice de la pluie (Cobo, 1964 [1653], liv. I : ch. VII). Illapa occupait une place aussi importante que Viracocha et le Soleil ; il était connu sous ce nom dans les Andes du Sud et sous celui de Libiac (Liviac, Lliviac) dans les Andes de I'ancien archidiocèse de Lima (Arriaga, 1999 [1621] : ch. II). C'est sur le sommet des montagnes que les fidèles l'imploraient et faisaient des sacrifices lorsque la sécheresse ou l'humidité menaçaient leurs récoltes (Métraux, 1983 : 114-116). Illapa, en tant que phénomène météorologique était toujours associé au tonnerre et à l'éclair. Certains grands centres cérémoniels à influence interrégionale étaient dédiés à une de ces représentations ; celle de Catequil chez les Huamachucos (province Sánchez Carrión) et Pariacaca chez les Yauyos (province de Huarochirí) (Castro de Trelles, 1992 : LIX). Il existait aussi des incarnations locales par exemple Yanaraman, aussi appelé Libiac Cancharco dans la région de Huánuco (Duviols, 1973 : 167). Dans la sierra, c'était le phénomène météorologique qui frappait le plus l'imagination qui était au cœur de leurs préoccupations : les tempêtes, les éclairs, le tonnerre ou la foudre, qui produisaient brusquement des ténèbres, des mugissements, et tuaient parfois de manière mystérieuse. Ce phénomène n'était pas la prérogative d'une seule divinité, il était toujours considéré comme provenant de plusieurs divinités ou comme leur attribut. Par exemple, dans un poème religieux inca, conservé par Garcilaso de la Vega, I'origine de la pluie et de la foudre est attribuée à une fille de sang royal : 
«Belle fille! Ton frère pluvieux rompt maintenant ta petite cruche; c'est pour cela qu'il tonne, qu'il y a des éclairs et que la foudre tombe. Toi, fille (de sang) royale, tu nous donneras par la pluie tes belles eaux; quelquefois aussi tu fais grêler sur nous et neiger de même. Celui qui a fait le monde, le Dieu qui l'anime, le grand Viracocha t'ont (?) donné une âme pour faire cette charge où ils (?) t'ont établie (...) » (Garcilaso de la Vega, 1967 [1609], liv. II : ch. XXVII) (ma trad.).

La fonction, la forme et le nom des êtres mythologiques sont donc des éléments qui varient selon les régions et au sein d'une même région, sans définir la nature des divinités ; ce qui rend difficile voire impossible leur identification.

Certaines populations de I'Intermédiaire récent, mais aussi les Incas, ont conféré à l'eau, sous la forme d'une lagune ou d'un lac, le statut de leur lieu d'origine (pacarina) qu'ils tenaient en très haute vénération. De fait, selon certains mythes incas, le lac Titicaca était le lieu des origines impériales de la noblesse inca, de son fondateur (ou héros culturel), et dieu principal : Viracocha. Sur les bords du lac, à Tiahuanaco, Viracocha, en tant que héros culturel, façonna, en terre ou en pierre, selon les versions, « différentes nations ». Il les envoya sous terre pour qu'elles émergent aux endroits qu'il leur avait désignés ; ces derniers étaient pour la plupart des montagnes, des lagunes et des sources. Cobo (1964 [1551], liv. I : ch. II) précise que les populations vénéraient ces endroits comme leur huaca principal, car elles pensaient qu'ils étaient leurs lieux d'origine.

À la lecture des divers ouvrages relatifs aux nettoyages des canaux d'irrigation dans la Sierra péruvienne (Borea, 2004 ; Farfán, 2002 ; Gelles, 2002 ; Gose, 1994 ; Isbell, 1978 ; Lecaros, 1996 ; Llanos \& Osterlings, 1981 ; Raez, 2001 ; Rivera, 2002 ; Tello \& Miranda, 1923 ; Valderrama \& Escalante, 1988 ; Vargas, 1990), il apparaît qu'actuellement l'eau est considérée comme un élément androgyne car elle englobe un principe de vie qui est tantôt associé au genre masculin tantôt au genre féminin. En tant que principe de vie masculin, l'eau est associée aux montagnes, aux pics enneigés, aux ancêtres fondateurs y résidant (apu ou wamani, ancêtres éloignés communs aux habitants de diverses communautés d'une région déterminée) qui sont considérés comme des divinités pourvoyeuses d'eau. Isbell (1978 : 139) et Ossio (1992 : 313-314) pensent que l'eau du dégel circulant dans les canaux d'irrigation est la semence du wamani qui agit comme fécondatrice de la terre. D'autres interprétations (Villar Córdova, 1933) suggèrent que les sommets enneigés et l'eau du dégel peuvent être associés aussi à l'action d'une divinité féminine, comme c'est le cas pour le pic de la Veuve de la vallée étudiée. L'eau comme principe de vie féminin est généralement associée à la mer, aux lagunes et aux sources ; même si, pour la plupart, elles déversent de l'eau provenant du dégel des pics enneigés voisins.

Vraisemblablement, malgré les siècles écoulés et les importants changements environnementaux et socioculturels qui ont affecté ces populations montagnardes, certaines croyances relatives à l'origine de l'eau d'irrigation semblent être enracinées dans celles de la période préhispanique et avoir persisté. Nous l'observons dans le rituel de la limpiacequia dans lequel entrent en scène des divinités pourvoyeuses d'eau ainsi que des êtres mythologiques qui leur sont associés.

\section{LA COMMUNAUTÉ DE SAN PEDRO DE HUACOS ET SON CALENDRIER AGRICOLE}

La communauté de San Pedro de Huacos, reconnue en tant que communauté paysanne le 5 septembre 1956, est située, à 3252 m d'altitude, dans la zone quechua de la rive droite de la vallée haute du Chillón (fig. 1). Au recensement effectué par CORDELIMA 


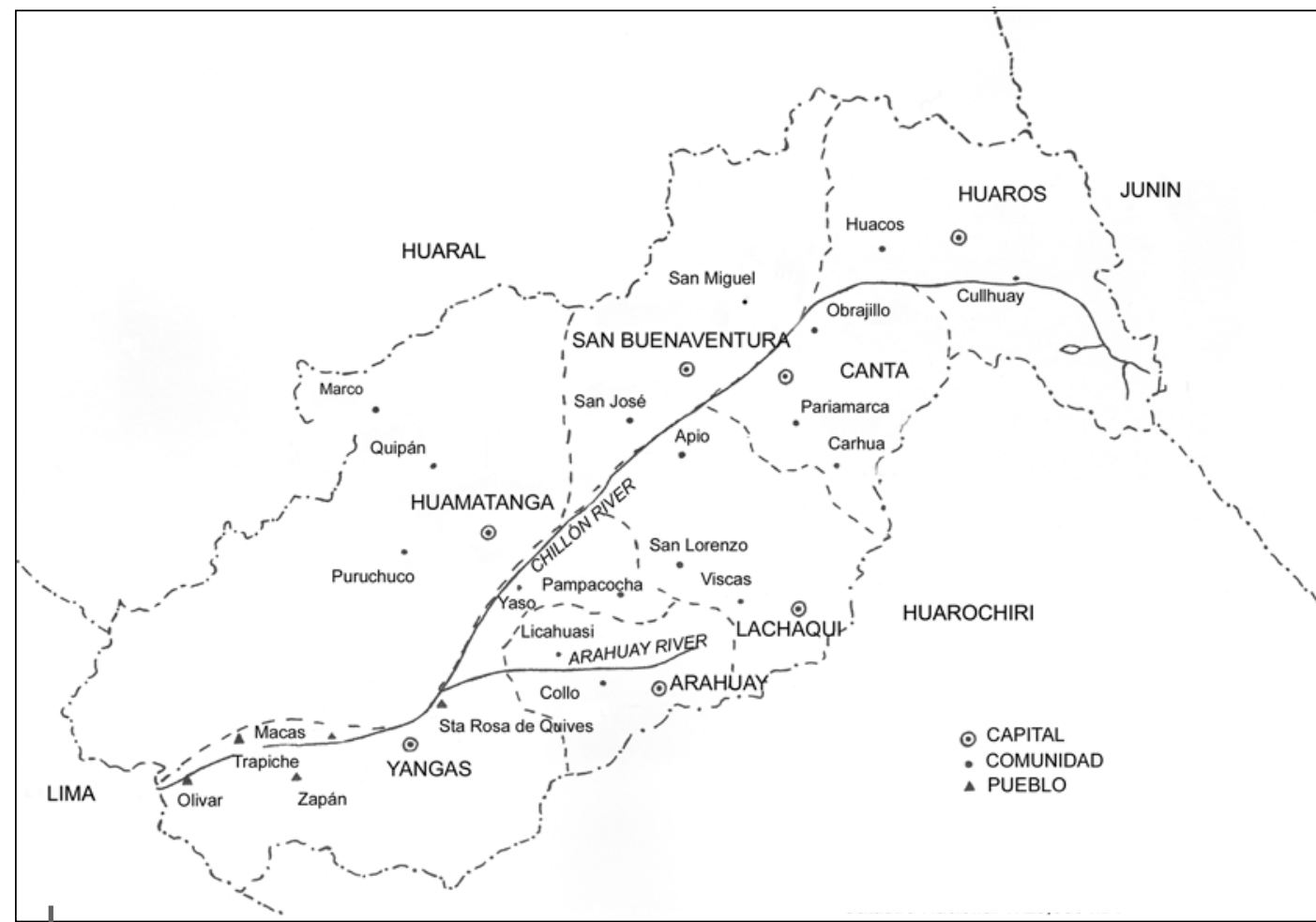

Figura 1 - Carte générale de la vallée du Chillón (province de Canta, département de Lima) (Silva, $1996:$ 440)

Échelle : 1: 25000 (redessiné d'après Ramírez, 1995)

Source : Carte nationale 1: 100000 ING

Cadastre national 1: $25000 \mathrm{MA}$

(Corporación Departemental del Desarrollo de Lima) en 1992, elle comptait 280 habitants. Actuellement, ce nombre a fortement diminué en raison de l'attraction croissante de la capitale située à $118 \mathrm{~km}$ et aux alliances matrimoniales inter et extracommunautaires qui ouvrent de nouveaux réseaux de rapports préférentiels. Nous estimons à 200 personnes le nombre de résidents et à environ 50 le nombre de comuneros calificados, c'est-à-dire des personnes qui ont le statut de comuneros (membres de la communauté). Ce sont les représentants des unités domestiques et souvent quelques adultes isolés (célibataires, veufs ou personnes séparées sans progéniture à charge), mais qui ne résident pas obligatoirement dans les limites territoriales de la communauté. Les terres de la communauté couvrent 6500 ha dispersées entre 2900 m et 5000 m d'altitude, dans les zones quechua, suni et puna, dont seuls 457 ha sont des terres cultivées, soit $7 \%$ de la totalité du territoire. Ces 457 ha englobent 252 ha de terres irriguées, 200 ha de terres de culture non irriguées et 5 ha de terres forestières (Huacos, fiche technique, Valcárcel \& Lumbreras, 1997 : 75). La communauté possède trois canaux qui irriguent deux secteurs différents : les terres moyennes et les terres basses, situées entre $2835 \mathrm{~m}$ et $3450 \mathrm{~m}$ d'altitude. Quant aux hautes terres, qui constituent le troisième secteur, elles dépendent uniquement de l'eau de pluie. La plupart des comuneros possèdent des terres dans les trois secteurs, ainsi ils ont accès à des denrées diversifiées produites par les différents paliers écologiques. Les cultures 
se composent de nombreuses variétés de tubercules, de maïs, de fèves, de luzerne, de blé et d'orge. Le canal d'Ongongoy irrigue les terres moyennes et les canaux de Shispo et de Mayura ${ }^{4}$ se partagent respectivement les basses terres du nord-est et celles du sud-ouest. Les canaux préhispaniques d'Ongongoy et de Shispo, faits de terre et de pierres, reçoivent leurs eaux de la lagune Cushurcocha via la vallée sèche d'Ongongoy (fig. 2).

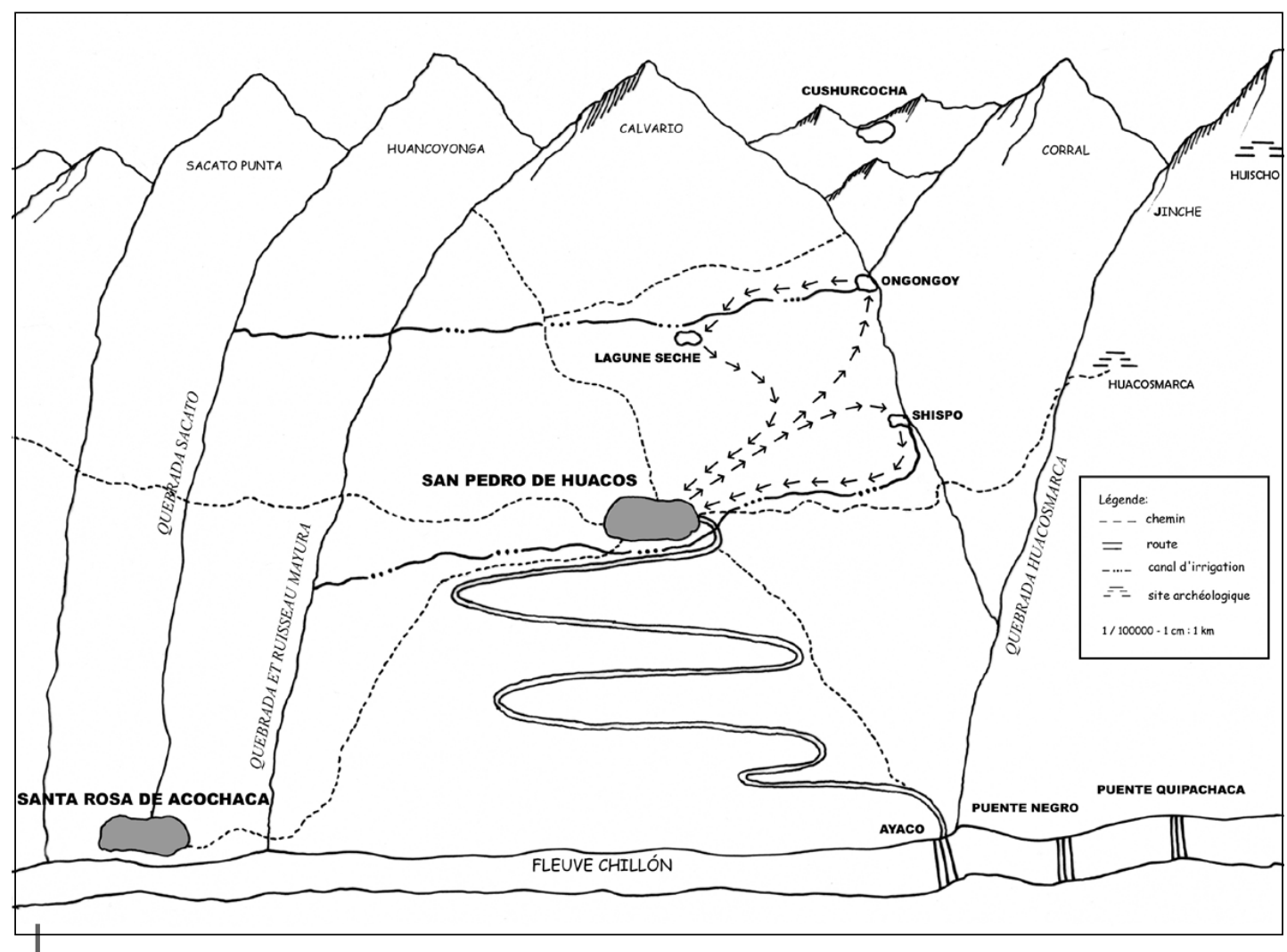

Figure 2 - Schéma du déroulement de la limpiacequia d'Ongongoy et de Shispo (dessin de l'auteur)

Le nettoyage rituel des canaux d'Ongongoy et de Shispo a lieu deux fois par an : en avril et en août. Seul celui réalisé le troisième dimanche du mois d'août ainsi que le samedi et le vendredi qui le précèdent fait l'objet d'une description.

Les observations participantes faites durant le rituel du mois d'août ainsi que les entretiens menés à sa suite, nous ont permis de remarquer qu'à San Pedro de Huacos le rituel est davantage intégré dans une structure calendaire agricole que dans un calendrier religieux. Notons en effet que le nettoyage du mois d'avril correspond à la fin de la saison des pluies et au début de la saison sèche (du mois d'avril à la mi-juin) et est associé avec les semailles des premières papamauay (pommes de terre cultivées grâce à l'eau d'irrigation), tandis que

4 Le canal de Mayura est un nouveau canal qui a été construit il y a quelques années pour palier les insuffisances en eau des terres situées à côté de la vallée sèche du même nom. Il ne fait l'objet d'aucune célébration. 
celui du mois d'août correspond à la pleine saison sèche 5 (de la mi-juin au mois d'août inclus) et est associé aux semailles d'autres tubercules : les ocas et les ollucos. Selon les témoignages des comuneros, les nettoyages des canaux d'irrigation précèdent toujours la première irrigation des terres. Celle-ci sert à ramollir la terre des champs et des canaux ; ce qui rend le labourage des terrains cultivés, la remise en état de certains canaux et les semailles, plus faciles. Pour ces périodes, le processus des activités agricoles (nettoyageirrigation-semailles-moissons) est donc similaire. Le nettoyage inaugure donc un nouveau cycle de croissance des terres ensemencées.

Cependant, malgré la forte connotation agricole du rituel6, il est considéré par les comuneros comme l'une des célébrations les plus importantes de l'année, avec celle de la fête patronale, car on y célèbre la Mamahuaca : la gardienne des eaux de la communauté. À notre sens, ce personnage mythique local fait partie de la catégorie des divinités pourvoyeuses d'eau d'irrigation dont nous avons mentionné l'existence et la vénération comme maîtresses et, pour la plupart, créatrices des canaux dès l'époque préhispanique.

\section{LA VERSION «ANCIENNE » DU RITUEL : DESCRIPTION}

L'ancienne version du rituel a été reconstituée à partir des témoignages des comuneros les plus âgés de Huacos. Elle se réfère donc à une période comprise entre la fin du XIXe siècle et le début du XX $X^{e}$ siècle. Le rituel se déroulait pendant trois jours. Chaque jour de la limpiacequia correspondait, et correspond d'ailleurs toujours, au nettoyage d'un canal ou d'un tronçon de canal situé dans un palier écologique particulier et généralement différent du précédent. La bonne réalisation du nettoyage et sa qualité étaient vérifiées par le répartiteur d'eau qui est l'autorité7 qui répartit, encore aujourd'hui, l'eau de la communauté et contrôle l'irrigation des terres cultivées. II menait la cadence du nettoyage en criant des numéros qu'il avait attribués auparavant à l'ensemble des comuneros. Ils représentaient l'ordre dans lequel les comuneros devaient se succéder pour nettoyer une distance spécifique appelée $t^{\circ}{ }^{8}$. Chaque journée était donc dédiée à la réalisation d'activités précises qui se déroulaient à des moments et à des endroits institués par la coutume, c'est ce que nous appelons la séquence rituelle.

La cérémonie bisannuelle de la limpiacequia est la seule activité agricole à rassembler, sur base d'un consensus communautaire, tous les membres de la communauté à un moment et en un lieu donnés. Soulignons que la participation obligatoire au nettoyage des canaux trouvait sa contrepartie dans l'accès de chaque participant à l'eau d'irrigation pour sa

5 Les mois mentionnés pour les deux périodes sont ceux du calendrier agricole de la communauté étudiée. Il est impossible de donner une estimation correcte d'un calendrier agricole général pour l'ensemble des communautés montagnardes péruviennes car, suivant leur situation dans un palier écologique spécifique, les saisons fluctuent de manière importante (de deux à trois mois).

6 En analysant ce rituel sur le terrain, nous avons remarqué que les comuneros s'y réfèrent en tant que fiesta del agua (fête de l'eau) et non en tant que faena ni faena hydraulique festive.

7 La situation de San Pedro de Huacos ne peut pas être généralisée aux communautés villageoises de la sierra. Il est, en effet, assez rare, que celles-ci possèdent uniquement un répartiteur d'eau. Pour la plupart, l'accès individuel à l'eau d'irrigation, en tant que ressource communale, est contrôlé par une assemblée regroupant les utilisateurs locaux. Celle-ci est appelée la comisión de regantes, la junta de regantes ou encore comité de regantes. Cette organisation trouve son origine dans le Código de Aguas de 1969 qui est un décret-loi de la réforme agraire dont une des caractéristiques était la mobilisation sociale du monde paysan, imposée autoritairement, dans le cadre d'associations populaires sous contrôle gouvernemental (Gelles, 2002 : 136).

8 Ancienne unité de mesure incaïque qui dans le cas de la communauté de Huacos équivaut à la longueur de deux pelles (Huacos, août 2004). 
parcelle. Ce système est encore d'actualité mais de nos jours, certains comuneros et notables qui résident à Lima ne se déplacent pas pour la célébration. Ils font alors face à une amende ou à une suppresion de leur tour d'irrigation pendant plusieurs semaines. Cependant, certains engagent un travailleur journalier qui, pour la somme de vingt soles, doit effectuer durant toute la cérémonie le nettoyage des canaux à la place de son employeur.

La version actuelle du rituel à laquelle nous avons assisté est mise en parallèle avec celle décrite dans le présent article ; nous y ferons référence pour souligner les changements significatifs majeurs dont nous ne pouvons faire l'économie pour mener notre analyse, mais aussi pour compléter les données concernant la séquence rituelle ancienne.

\section{1. La séquence rituelle du vendredi}

Le vendredi à l'aube, avant de commencer le nettoyage du tronçon du canal d'Ongongoy, qui va du lieu-dit la « lagune sèche » à la vallée sèche de Mayura, une petite commission, composée de personnages rituels appelés les parianes, se dirigeait vers la chute d'eau d'Ongongoy pour faire des offrandes de coca, d'alcool, de cigarettes et de sang de cochon d'Inde à la Mamahuaca. Les personnages des parianes, parés d'une peau de renard ou d'une peau de puma, étaient joués par les ministriles 9 de la communauté, et plus particulièrement les jeunes alguazils. Durant les limpiacequias, ils étaient considérés comme les aides du répartiteur d'eau mais aussi comme des intermédiaires privilégiés entre la Mamahuaca et les comuneros. Selon les Huacosinos (habitants de Huacos) les plus âgés, la Mamahuaca, de teint pâle et d'apparence étrangère vivait, et vit toujours, dans la chute d'eau d'Ongongoy. Elle était vêtue de blanc et portait une couronne en or sur la tête.

À la fin du XIXe siècle et au début du XXe, la Mamahuaca était confondue avec le lieu naturel de la chute d'eau d'Ongongoy où elle était censée résider. Grâce aux données des entretiens avec les plus anciens, nous pensons qu'elle était aussi confondue avec l'eau d'irrigation elle-même ou alors, tout au moins, qu'elle avait une certaine influence sur son débit. Car si les parianes ne réalisaient pas les offrandes à la chute d'eau, c'est-àdire la Mamahuaca, les comuneros affirment que l'eau d'irrigation venait à manquer pour le reste de l'année agricole. C'est sans doute pour cela qu'actuellement, lorsque certains comuneros se rendent sur leurs champs situés à proximité d'Ongongoy, ils emportent des feuilles de coca et des cigarettes qu'ils offrent à la Mamahuaca avant d'irriguer leurs parcelles de terre.

Une fois les offrandes à la Mamahuaca faites par les parianes, le nettoyage du tronçon du canal d'Ongongoy pouvait commencer. Pendant ce temps, les parianes se rendaient à la lagune sèche pour garnir sa surface intérieure de paille séchée, appelée ichu, récoltée près des lagunes de la puna.

Selon la plupart des comuneros, le déroulement du nettoyage ne présentait pas de différence majeure avec celui d'aujourd'hui. Un changement est pourtant notable concernant les acteurs de la séquence rituelle. Actuellement les alguazils ne jouent plus le rôle des parianes et ces derniers ne sont plus représentés dans la séquence rituelle; ce qui entraîne immanquablement la suppression des offrandes faites à la Mamahuaca. Les plus

9 Le terme ministriles désigne les membres du conseil exécutif de la communauté qui ont à leur charge les fonctions d'inspecteur, de régisseurs, de campos et d'alguazils. Actuellement les ministriles occupent une fonction de protection, de sécurité et de vigilance pour que perdure l'ordre au sein de la communauté. Les directives leur sont données par les autres autorités du conseil exécutif et par d'autres autorités politiques, telles que le juge de paix ou le lieutenant-gouverneur. 
anciens de la communauté, qui ont pour la plupart joué le rôle de ces personnages rituels, l'expliquent par le fait que les jeunes comuneros ne sont plus intéressés par la continuation de cette coutume ancestrale que les anciens font remonter à l'époque incaïque. Le parian est en effet déjà mentionné au XVIIe siècle dans l'écrit d'Arriaga (1999 [1621] : ch. II) et la chronique de Guaman Poma de Ayala (1987 [1615] : foj. 860[874], 1140[1150], 1141[1151]). Les deux hommes le présentent comme un personnage vêtu d'une peau de renard qui avait la charge de garder les terres cultivées et d'effrayer les animaux (ou les voleurs) qui venaient manger les semences et les fruits mûrs. Selon Guaman Poma de Ayala (1987 [1615] : foj. 803[817]) cette charge, d'origine incaïque, était celle des jeunes alguazils de l'époque coloniale.

Vraisemblablement à San Pedro de Huacos, à la fin du XIXe et au début du XXe siècle, les alguazils occupaient toujours le rôle des parianes mais leur fonction n'était plus celle de gardiens des cultures. À cette époque, dans la communauté de Huacos, comme dans d'autres communautés de la zone étudiée, il apparaît comme un personnage rituel, sous la forme d'un renard (ou d'un puma), associé aux divinités pourvoyeuses d'eau10. Borea (2004), dans un article dédié à l'évolution ethnohistorique de ce personnage, explique la mutation de la fonction du parian par le changement des institutions sociales et politiques. Selon Guaman Poma de Ayala, le parian joué par les alguazils dépendait des autorités traditionnelles, los varayos. La disparition quasi totale de ces autorités aurait amené la disparition du rôle de gardien des cultures qui se serait mué en charge rituelle dans le cadre des limpiacequias de la vallée haute du Chillón mais aussi dans celles d'autres communautés de la cordillère de Lima.

\section{2. La séquence rituelle du samedi}

Les données de la séquence rituelle ancienne de cette journée ne sont compréhensibles qu'à la lumière de celles de la séquence rituelle actuelle. Pour décrire l'ancienne version du rituel du samedi nos informateurs l'ont constamment comparée à la version actuelle en soulignant les changements qui l'ont affectée.

De nos jours, la journée du samedi est considérée comme le jour central du rituel et le moment culminant de la séquence rituelle prend place à l'extérieur de la communauté lors de la table rituelle. Le samedi est caractérisé par la division en deux groupes ${ }^{11}$ de l'ensemble des comuneros et des visiteurs : le groupe des mariés et le groupe des célibataires, luimême divisé en deux sous-groupes : le groupe des solteros (célibataires de sexe masculin) et celui des solteras (célibataires de sexe féminin). Les autorités effectives du rituel actuel sont les mayordomos 12 (les chargés de fête). Ils sont au nombre de trois : le chargé de fête du groupe des mariés, celui du groupe des solteros et celui du groupe des solteras. Chaque chargé de fête est accompagné d'une chargée de fête. Ils ont la fonction d'organiser le rituel et de gérer les dépenses qui s'y rapportent. De plus, ils ont la charge de vénérer, et célébrer

10 Son rapport à l'eau est aussi visible par les herbes (paille ou ichu) qu'il transporte avec lui. Gose (1994 : 98) a en effet démontré que la paille utilisée lors des nettoyages de canaux de la région d'Ayacucho était utilisée en tant que symbole des sources d'eau des hauteurs et des lagunes.

11 Le groupe des mariés est composé uniquement de comuneros et de quelques visiteurs mariés. Tandis que les personnes formant le groupe des solteros ne sont pas obligatoirement des comuneros ; il est généralement composé de jeunes, hommes et femmes, venus de Lima pour assister à la « fête de l'eau ».

12 Dans la plupart des écrits anthropologiques, le terme mayordomos se réfère aux autorités responsables de l'organisation de la fête patronale. Les habitants de San Pedro de Huacos différencient pourtant ce terme de celui appliqué aux autorités du nettoyage rituel. Les autorités de ces deux célébrations sont d'ailleurs différentes. 
les trois croix de la communauté. Durant la matinée, dans trois maisonnées différentes, les mayordomos ont l'obligation d'offrir le petit déjeuner à l'ensemble des personnes présentes pour le rituel.

Ensuite vers midi, accompagnés du juge de paix portant une bannière blanche à la main, ils ouvrent la marche vers la chute d'eau d'Ongongoy où a lieu le baptême par aspersion de ceux qui s'y rendent pour la première fois. Cet arrêt est la première halte de la célébration du samedi qui est caractérisée par la division spatiale des participants : le groupe des célibataires se situe à côté de la chute d'eau tandis que le groupe des mariés se trouve à côté du canal d'Ongongoy. Les deux groupes effectuent leurs célébrations respectives pendant 2 heures. L'ambiance du côté des célibataires est festive, tous dansent et chantent sur les airs d'un orchestre en buvant de la chicha (bière de maïs fermenté) et de la bière. Du côté des mariés, seuls les sons de la harpe et du violon brisent le silence solennel des mariés en train de mâcher la coca, de fumer les cigarettes et de boire l'alcool (fig. 3). Le groupe des mariés, sous I'œil attentif du répartiteur, commence rapidement à nettoyer la partie du canal qui va de la chute d'eau à la lagune sèche en faisant une seconde halte à Cunturquishe qui se situe le long des canaux à mi-chemin entre la chute d'eau et la lagune sèche.

La division communautaire en deux groupes distincts ainsi que la charge des mayordomos, comme nous la connaissons actuellement, n'existaient pas dans l'ancienne séquence rituelle. La division est advenue car les jeunes ne se sentaient pas concernés par la célébration des mariés ; ils ont donc décidé de faire une autre célébration plus festive. Naguère, seuls les ministriles, jouant le rôle des parianes, et le répartiteur d'eau étaient les plus hautes autorités du nettoyage rituel ; la fonction du mayordomo n'existait pas encore. C'était le répartiteur d'eau qui jadis, avec une partie du salaire versé par les comuneros, était chargé des dépenses de la cérémonie : la rémunération d'un joueur de chirisuya13, de deux veuves chanteuses et joueuses de petit tambourin et l'achat de fleurs, de la coca, de cigarettes et d'alcool.

On remarque que les dépenses effectuées autrefois par le répartiteur sont similaires à celles encourues actuellement par les mayordomos, à l'exception des dépenses relatives aux musiciens qui ne font plus partie des acteurs du rituel. Au fil des années la charge du répartiteur d'eau, ainsi que celle de ses aides est devenue de moins en moins importante au profit de celle des mayordomos. Néanmoins, de nos jours, dans l'esprit des Huacosinos, c'est le répartiteur qui est l'organisateur et l'autorité principale du rituel de nettoyage. Ils préfèrent d'ailleurs la dénomination " parrains des croix » pour les chargés de fête plutôt que le terme mayordomos.

Continuons la description en parallèle des versions du rituel.

Dans la version actuelle, les deux groupes, mariés et célibataires, se rejoignent et se confondent au lieu-dit Cunturquishe qui est la seconde halte. Pendant près d'une heure, tous les participants y dansent et chantent au son des deux ensembles musicaux.

Dans la version ancienne, la population effectuait une halte à Cunturquishe afin d'élire les nouveaux parianes parmi les jeunes alguazils. L'un d'entre eux devait se parer d'une peau de renard et un autre d'une peau de puma. Dès l'élection terminée, deux des parianes avait la charge d'aller fermer l'arrivée d'eau du canal d'Ongongoy.

Actuellement, après la deuxième halte à Cunturquishe, les autorités du rituel (les mayordomos, le répartiteur d'eau, le vice-président de la communauté et le juge de paix)

13 La chirisuya est un aérophone à anche double caractéristique des pays d'Amérique du Sud et plus particulièrement du Pérou. Il fait partie du groupe d'aérophones à anches doubles de l'Amérique espagnole regroupé sous le terme générique de Chirimías. Selon Chamorro (1982 : 174-177), il fut probablement introduit par les Espagnols dans la liturgie catholique, dès le dernier tiers du XVIIle siècle, afin d'encourager les Indiens à la conversion. Son origine est incertaine mais le système d'embouchure évoquerait une influence des instruments à vent mauresques. 

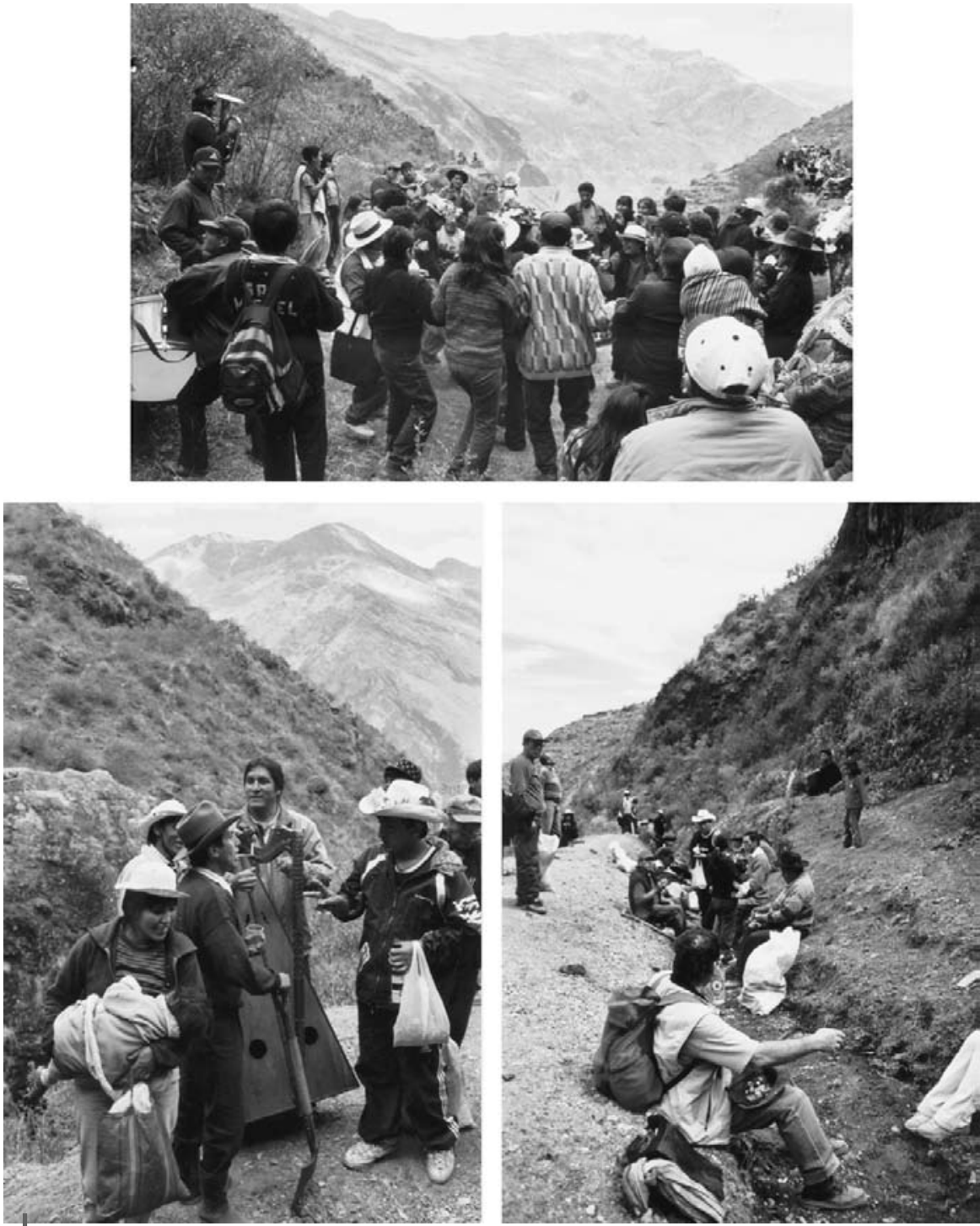

Figura 3 - Les célébrations des célibataires (haut) et des mariés (bas)

(photographies de l'auteur)

partent les premiers vers la lagune sèche. Ils sont reconnaissables par la ficelle, hualga ${ }^{14}$, qu'ils portent autour de leur torse et qui supporte divers aliments (piment, pomme de terre, pain, tomates) (fig. 4). Tant que le juge de paix n'a pas planté la bannière et prononcé

${ }^{14}$ Cet ensemble de denrées est appelé colgaje dans la communauté de Lachaqui et qalqinchas dans la communauté de Cabanaconde (département d'Arequipa, province de Caylloma) (Gelles, 2002 : 199 ; Raez, 2001 : 343). Il semble qu'ils soient portés par les personnes qui parrainent de grands événements rituels. Ces ensembles sont peut-être le symbole de l'abondance souhaitée. 


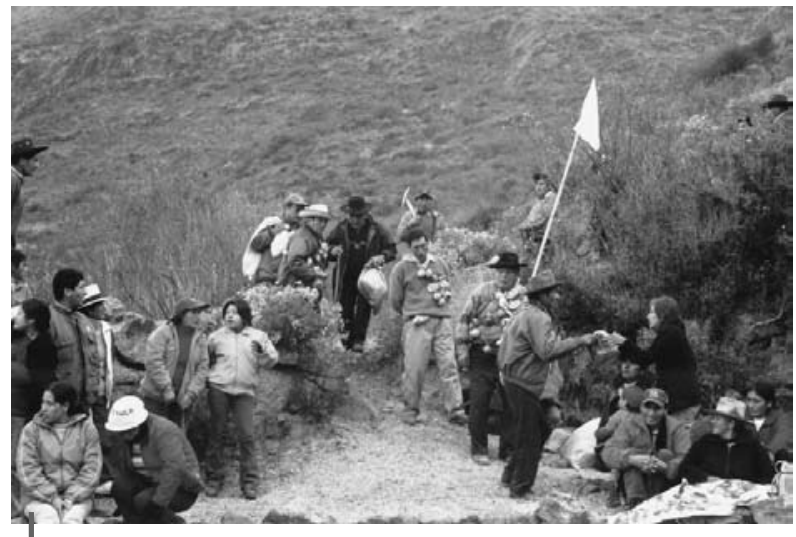

Figure 4 - Arrivée à la lagune sèche des autorités portant le hualga (photographies de l'auteur) le discours d'hommage aux ancêtres considérés comme les créateurs des canaux de la communauté, seules les autorités peuvent fouler le sol du centre de la lagune; le reste des comuneros se contente d'attendre la permission de pouvoir s'y asseoir pour débuter la table rituelle. Dès la fin du discours chacun installe sa nappe et ses plats à l'endroit qui lui est désigné par la coutume. S'ensuivent des échanges incessants de nourriture et de boissons. II règne une ambiance totalement chaotique autour de la table rituelle.

Une heure plus tard, le soleil s'est couché et les comuneros, à la seule lueur de la lune, redescendent en dansant et en chantant jusqu'à la place centrale de la communauté où la fête, arrosée de chicha et d'alcool, continue jusqu'aux petites heures du matin.

Anciennement, lors de la table rituelle, les parianes, certains déguisés en renard ou en puma, empêchaient les comuneros de manger en venant voler leur nourriture. Sur le chemin du retour vers la communauté et à l'intérieur de celle-ci, un autre type de rapt avait lieu. Les parianes enlevaient les enfants en bas âge et les emportaient dans une petite maison située sur les hauteurs de la communauté. Leurs parents venaient les sauver en les échangeant contre certaines denrées alimentaires (des fèves, des pattes et de la viande de boeuf, du blé, des haricots et du maïs) ou parfois de l'argent. Pendant ce temps d'autres parianes, parcourant la communauté, demandaient des vivres à chaque chef de famille. En fait, les chefs des unités domestiques, de même que les parents des enfants, leur donnaient volontiers les vivres désirés car ils étaient destinés à la préparation du patache. Le patache était, jusqu'il y a une vingtaine d'années, le plat traditionnel des limpiacequias de la vallée haute du Chillón. La nuit même, sur la place centrale, les veuves de la communauté avaient l'obligation d'en commencer la préparation pour le lendemain après-midi. La cérémonie était accompagnée d'airs joués par le joueur de chirisuya et les joueuses de petit tambourin.

\section{3. La séquence rituelle du dimanche}

Comme pour la description du samedi, nous reprendrons les données de la version actuelle du rituel pour compléter celles de la version ancienne. Le dimanche clôture le rituel du nettoyage des canaux de la communauté de San Pedro de Huacos.

Actuellement, les comuneros considèrent la limpiacequia de Shispo comme secondaire. Ils ne voient pas l'utilité de s'y rendre car la plupart ne possèdent plus de terres cultivées dans cette zone. Ce sont surtout les jeunes gens venant de Lima pour le week-end qui y vont pour continuer la célébration du jour précédent. Certaines autorités s'y rendent aussi : le vice-président, le répartiteur d'eau et les mayordomos des croix des mariés et des célibataires. Vers midi, les autorités, les comuneros et les jeunes, encore ivres de la veille, partent en direction du canal. Arrivés au canal, le répartiteur ferme une vanne située dans les hauteurs de cette première halte. Pendant ce temps, deux personnes distribuent la coca et l'alcool aux comuneros et aux visiteurs, tandis qu'un joueur de harpe anime les danses. 
Le nettoyage du canal en direction de la communauté est effectué, vers 16 heures, par six comuneros. Ils sont accompagnés des jeunes qui dansent et boivent à côté du canal. Le nettoyage se termine vers 19 heures et tous rentrent en dansant vers la place centrale de la communauté. Arrivés à la place, tous se séparent et rentrent chez eux rapidement.

Anciennement, le dimanche était le jour principal du rituel et la « danse des parianes », qui avait lieu à l'intérieur de la communauté, était le point culminant de la séquence rituelle, alors que dans la version actuelle du rituel, il s'agit du samedi avec la table rituelle à la lagune sèche.

Les comuneros ne nous ont pas expliqué l'ancienne séquence rituelle de la limpiacequia de Shispo. Tous se sont attardés à décrire les événements qui se déroulaient à l'intérieur de la communauté.

Seuls les hommes se rendaient au nettoyage du canal, tandis que les femmes, les enfants et les parianes restaient dans la communauté. Sur la place principale, les veuves continuaient la préparation du patache qu'elles faisaient cuire dans d'énormes marmites. Pendant ce temps, les parianes disposaient des branches pour former un grand cadre qu'ils remplissaient d'ichu pour exécuter ce que les Huacosinos appellent « la danse des parianes ». Pour la circonstance, ceux-ci portaient une couronne d'ichu sur la tête, deux châles anciens de taille différente et un bâton dans la main. Le parian jouant le rôle du puma avait la tête recouverte d'une peau de puma et tenait, à la main, une patte séchée de vache. Le parian qui jouait le rôle du renard portait une peau de renard comme couvre-chef et tenait à la main, une patte séchée de mouton. À l'intérieur du cadre d'ichu, ils couraient derrière les autres parianes. En fait, il s'agissait plus de compétitions sous forme de jeux entre les parianes et les membres de la communauté, que de danses au sens strict.

Vers 15 ou 16 heures, les comuneros ayant fini de nettoyer le canal de Shispo, rentraient pour assister au spectacle donné par les parianes. Une personne désignée au préalable, portant la bannière blanche du juge de paix, marchait en tête. Les parianes avaient I'obligation de lui enlever la bannière. S'ils ne parvenaient pas à s'en emparer, on pensait que les récoltes seraient mauvaises. Les veuves qui cuisinaient le patache étaient les alliées des parianes ; pour les aider, elles jetaient du charbon encore tiède sur les comuneros. Après s'être emparés de la bannière blanche, les parianes la plaçaient en haut de la tour de l'église ; ce qui signifiait que la communauté avait retrouvé la sérénité.

Avant la consommation générale du patache, un dernier jeu avait lieu sur la place principale. L'ensemble de la communauté se divisait en deux groupes, l'un était celui du renard et l'autre celui du puma. On traçait une ligne au milieu de la place et les deux groupes s'affrontaient en tirant sur une corde. Celui qui arrivait à faire passer le groupe adverse de son côté de la ligne était reconnu vainqueur. Lorsque le groupe des pumas gagnait, on pensait que les récoltes seraient mauvaises et que dans le cas inverse, elles seraient favorables. Finalement, tous les comuneros et les parianes se retrouvaient sur la place centrale pour manger le patache, ce qui concluait la limpiacequia de la communauté.

\section{ANALYSE DE LA SÉQUENCE RITUELLE}

La confrontation des descriptions de la limpiacequia dans sa version ancienne et dans sa version actuelle, nous montre une évolution diachronique particulièrement importante en ce qui concerne les acteurs et les pratiques. Seuls l'ordre et la durée du nettoyage des canaux ne semblent pas avoir été modifiés, soit d'abord le nettoyage du canal d'Ongongoy pendant deux jours suivi de celui de Shispo pendant une journée. 
Pourtant, le travail communautaire subsiste. La participation des comuneros est certes une obligation mais elle est aussi un privilège puisqu'en termes de droit coutumier andin, elle sert à déterminer et à réaffirmer les droits des irrigateurs sur les eaux et sur les canaux de la communauté, et par là même leur appartenance à la communauté ; et cela tout au moins depuis l'époque inca (Sherbondy, 1979 : 58 ; 1987 : 131). De manière idéale, le droit d'utilisation des acequias est fonction de l'appartenance des comuneros à une ascendance commune. À Huacos, les ancêtres fondateurs et la Mamahuaca étaient, et sont toujours, considérés comme les constructeurs d'Ongongoy et de Shispo. Actuellement, d'ailleurs, le juge de paix les évoque et les mentionne le samedi à la lagune sèche. Salomon (1997 : 266) conclut que nous assistons dans le cadre communautaire à une mobilisation rituelle de la mémoire collective pour la reproduction des droits villageois et des structures d'autorité liée à l'irrigation. La persistance de ce rituel est également tributaire de l'histoire particulière des sociétés andines. Plusieurs américanistes (Condori \& Gow, 1976 ; Sallnow, 1987 ; Urton, 1981) s'accordent en effet pour dire que ce sont les rituels agraires locaux actuels (dans le champ domestique, familial et parfois communautaire ou régional), tels que ceux de la limpiacequia et du marquage du bétail qui servent de fondement à l'ordre social autochtone et qui sont à charge des chefs de famille, de représentants de la communauté villageoise (ex. mayordomos), et parfois également de spécialistes (ex. parianes), qui ont le mieux résisté aux assauts des hommes et au temps car les concepts qui les fondaient et qui en découlaient étaient trop profondément enracinés dans les systèmes de pensée andins pour en être « extirpés » (Duviols, 1977). En outre, on peut attribuer cette « survivance» au fait que les rituels agraires fonctionnaient de manière autonome et qu'ils étaient très éparpillés et donc difficilement contrôlables par la hiérarchie catholique. Finalement, la religion chrétienne n'a pas fourni de soutien rituel alternatif aux pratiques agropastorales.

Notre propos n'est pas de présenter une cérémonie d'origine préhispanique non modifiée par le temps, ni d'affirmer que sa fonctionnalité et sa structure sont identiques à celles du rituel qui avait lieu durant les périodes récentes. Nous pensons que la religion des populations rurales andines actuelles de même que les valeurs morales qui s'y rattachent doivent être vues comme le produit à la fois de créations locales, insérées dans une synthèse « autorénovante » et dynamique d'apports multiples, et d'impositions liées aux processus préhispaniques d'unification (expansion inca) auxquels s'est ajouté l'impact de plusieurs siècles de colonisation espagnole (Malengreau, 1995 : 241-245).

Il est donc impossible d'appréhender le rituel comme une simple continuité à travers les siècles. Actuellement, par exemple, outre l'évidente nécessité d'avoir des récoltes abondantes grâce au meilleur écoulement de l'eau d'irrigation, les autorités et les plus anciens de la communauté entrevoient la réalisation du rituel comme une manière de valoriser cette coutume qu'ils attribuent à leurs ancêtres ; et à travers elle, de contribuer à la notoriété de la communauté à un niveau provincial. La renommée de la limpiacequia d'Ongongoy-Shispo, et donc de San Pedro de Huacos, est en effet tributaire de son bon déroulement et du nombre de participants. Ces derniers viennent généralement des communautés voisines ou parfois de régions plus éloignées comme celles du Cuzco ou de Jauja. En fait de nos jours, les comuneros se réapproprient les signes, les symboles ou encore les croyances de l'ancienne version du rituel pour produire un nouveau marqueur identitaire. Même si les parianes ne sont plus présents, les personnes âgées qui participent encore aux rituels ne manquent pas une occasion de les mentionner et de conter leurs exploits aux plus jeunes.

Ce rituel fait donc partie de la « pensée pratique » des comuneros de Huacos comme héritage qui peut être constamment recréé, manipulé et adapté afin de faciliter leurs relations avec le monde qui les entoure. Il est également difficile de discerner quelles 
sont les composantes d'origine préhispanique ou hispanique ou le degré syncrétique du phénomène actuel. Néanmoins, à la lecture comparative des données ethnographiques récoltées sur la version ancienne du rituel et celles provenant des chroniques coloniales, un concept, ancré au centre des représentations cosmogoniques du monde andin rural, apparaît : l'existence et la vénération de divinités pourvoyeuses d'eau et d'animaux mythiques qui leur sont associés.

L'idée d'une prolongation relative de la religion préhispanique, qui sous des formes locales et rurales particulières aurait intégré et réinterprété certains éléments chrétiens, est défendue par de nombreux anthropologues, et notamment Ossio (1992) et Ortiz (1973). Malengreau (1995 : 241) confirme d'ailleurs que :

« Une tradition autochtone semble en réalité constituer l'armature religieuse principale dans les Andes rurales centrales et méridionales de l'ancien Empire inca, et cela, soit sous une forme camouflée derrière des représentations et des pratiques religieuses catholiques, soit de manière parallèle aux expressions de la religion catholique, sans pour autant exclure la réinterprétation syncrétique ».

\section{LA NATURE FÉMININE DE LA DIVINITÉ POURVOYEUSE D'EAU D'IRRIGATION}

Le propos de l'analyse est de montrer l'influence des contextes historiques et culturels de la vallée haute du Chillón sur le développement d'une pensée autochtone propre.

Selon Rostworowski (1978 : 18, 156), durant la période pré-incaïque, la rive droite de la vallée du Chillón appartenait à la seigneurie des Atavillos. Leur domaine s'étendait le long de la vallée voisine de Chancay et était divisé en bas et haut Atavillos. Elle pense que le « grand chef » des Atavillos gouvernait non seulement sa propre seigneurie (chefferie ?) mais aussi celles de ses voisins: les Canta, Huamantanga, Piscas et Socos, qui régissaient à leur tour plusieurs ayllus (groupes lignagers de base) (fig. 5). La situation de la rive gauche du Chillón semble avoir été plus complexe. Une partie de la vallée haute appartenait à la seigneurie des 7 Guarangas (unité incaïque de 1000 tributaires) de Huarochirí qui occupait les régions montagneuses de Canta à Cañete et les vallées moyenne et basse appartenaient à la seigneurie de Colli (depuis la zone de Quivi jusqu'à la mer). Elle était délimitée par des bornes naturelles marquant la limite avec la seigneurie (chefferie ?) des Canta qui, elle, occupait la même rive de la vallée haute, depuis l'actuelle Santa Rosa de Quives jusqu'à la naissance du fleuve Chillón dans la Cordillère de la Veuve. Il est démontré que ces différentes populations entretenaient des rapports socio-économiques et des alliances temporaires (Justicia 413 Probanza de Canta, 1559, fol. 206. In : Rostworowski, 1988). II semble que durant l'époque pré-incaïque, existait une hiérarchie entre les seigneuries qui se partageaient la vallée du Chillón et de Chancay mais nous ne savons pas exactement comment fonctionnait cet ensemble de seigneuries. Formaient-elles une confédération socio-économique ou politico-militaire ou s'agissait-il d'une alliance temporaire entre groupes possédant une identité ancestrale commune ? La question reste entière.

Cependant Villar Córdova (1933), qui apporta I'une des premières contributions majeures aux recherches effectuées dans la vallée du Chillón ${ }^{15}$, émet, sur la base de données

15 Les données récoltées par Villar Córdova, et notamment la restitution du récit mythique, sont pour certains quelques peu douteuses. Son argumentation est certes uniquement basée sur la tradition orale de quelques communautés de la vallée haute du Chillón. Nous pensons néanmoins qu'elles peuvent servir à éclairer l'analyse entreprise dans le présent article. 


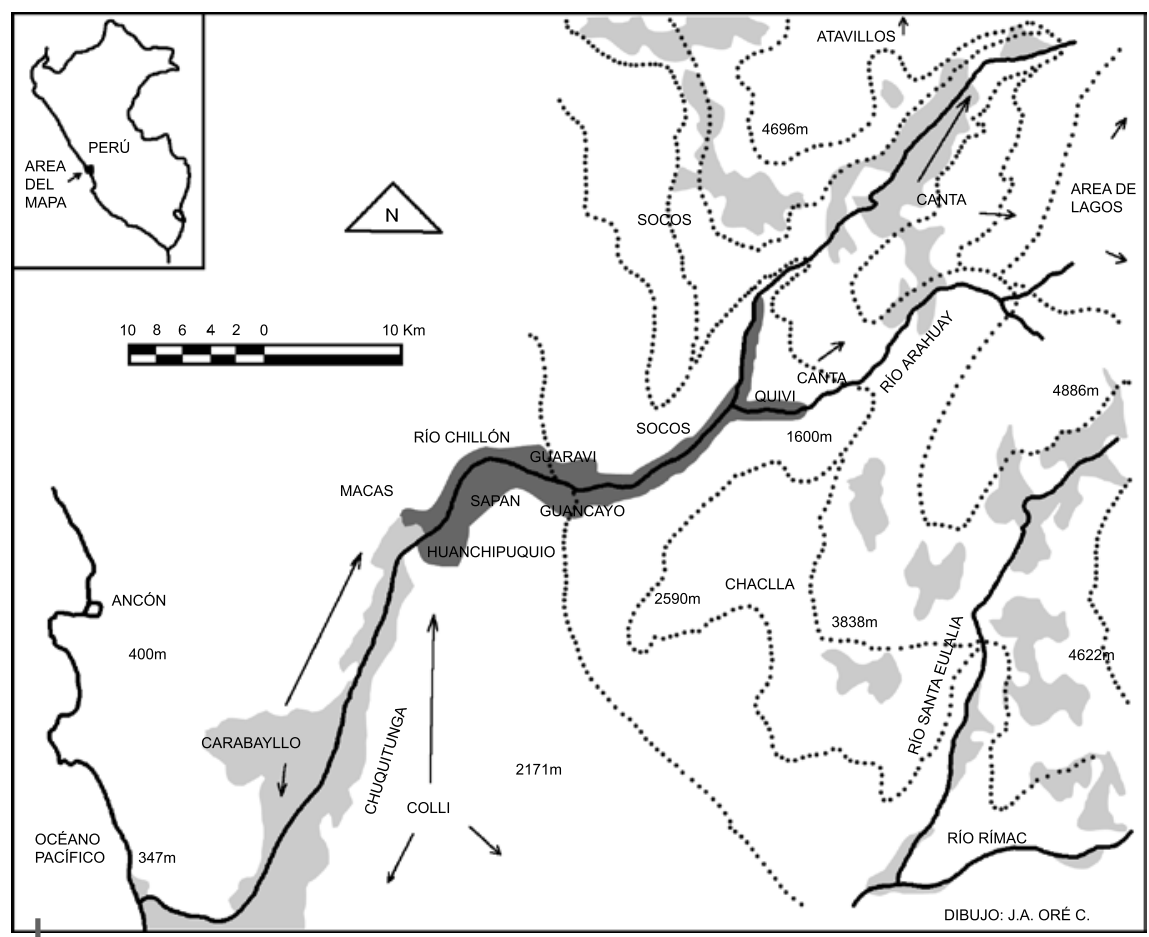

Figure 5 - Les seigneuries de la vallée du Chillón (redessiné d'après Dillehay, 1980 : 125)

ethnohistoriques, une hypothèse intéressante grâce au mythe des willkas recueilli dans la communauté de Cullhuay, située à proximité de la Cordillère de la Veuve. Nous en présentons ici un court résumé : Pachamama y est présentée comme l'épouse veuve de Pachacamac après que celui-ci se soit jeté dans la mer. Elle a de lui deux jumeaux de sexe différent, les willkas. Les deux orphelins et leur mère errent dans la nuit, perdus, et sont hébergés par le démon cannibale Wa-Kon. Celui-ci tente de séduire Pachamama et, devant son refus, la tue et la dévore. Les jumeaux willkas fuient et sont recueillis par une mouffette qui les nourrit de son sang et les aide à se venger de Wa-Kon en l'entraînant dans un piège où il trouvera la mort. Les willkas montent ensuite au ciel grâce à leur père qui les attendait. La fille se transforme alors en Lune et le garçon en Soleil ; la Pachamama est ressuscitée et récompensée de sa fidélité par Pachacamac qui lui confère la faculté génératrice.

Villar Córdova (1933 : 173-174) affirme que le pic enneigé de la Veuve était considéré par les populations locales de l'Intermédiaire récent, et nous supposons aussi par celles de I'Horizon récent (1450-1532), comme une grande divinité protectrice car c'est à cet endroit que la déesse Pachamama, en tant que veuve de Pachacamac, serait restée enchantée ; ce qui expliquerait d'ailleurs le nom de la cordillère. Selon lui, ce pic enneigé était un temple naturel à ciel ouvert, sans construction, ni architecture religieuse ; il était l'apu le plus vénéré de la région. II ajoute que ce sommet était la pacarina des Atavillos de Canta (il s'agit ici des anciens habitants de la rive gauche de la vallée haute du Chillón), et plus particulièrement des habitants de la zone de l'actuelle communauté de Cullhuay (rive droite) qui sont les plus proches de ce pic. Il précise que du temps où il mena sa recherche, cette communauté rendait toujours hommage aux divinités mentionnées dans le mythe, 
et plus particulièrement à Pachamama, sous sa dénomination de Veuve, lors des fêtes des récoltes, des semailles et du nettoyage des canaux d'irrigation.

En conclusion, Villar Córdova voit Pachamama, matérialisée par le Pic de la Veuve et célébrée en tant qu'ancêtre commun lors de cérémonies régionales et locales à caractère propitiatoire par les populations préhispaniques récentes de la vallée, comme l'élément cohésif probable des alliances faites par ces différentes populations ou seigneuries. De plus, grâce à l'exemple du nettoyage des canaux de la communauté de Cullhuay, il postule une continuité de la pratique rituelle à l'égard de la Terre-mère nourricière : Pachamama. Malgré l'argumentation rapide et la rédaction insuffisamment circonstanciée de son article son hypothèse semble fondée ; d'ailleurs notre étude ainsi que nos données de terrain la corroborent. En outre nous poussons l'analyse plus loin, en proposant que l'actuelle vénération de la Pachamama, sous la forme de divinités féminines locales, par les communautés du haut Chillón, trouve son origine dans l'influence côtière du site monumental de Pachacamac (vallée du Lurín).

Tout d'abord, I'association des divinités Pachacamac et Pachamama semble relever de deux évidences. La première découle de la nécessité pratique et idéologique d'expliquer aux populations du haut Chillón, à travers leur paysage mythique, la formation de deux astres célestes. À l'instar de la plupart des contes populaires actuels à caractère cosmogonique et étiologique, le mythe des willkas faisait sans doute partie à l'origine d'un ensemble plus large de récits destinés à détailler la formation de l'univers (Morote Best, 1988 : 78-97). Notons que sa structure est fort similaire à celle de la légende de Koniraya Viracocha présentée dans le manuscrit de Huarochirí (cf. Villar Córdova, 1933 : 161 ; Eeckhout, 1999 : 386) ; ce qui amènent certains auteurs à supposer l'existence de sens, de significations et de portées communes dans ces récits. La seconde évidence de cette association s'explique par les caractères complémentaires de ces deux divinités. Aux époques récentes, dans la conception dualiste andine de l'espace et de la société, caractérisée par deux moitiés opposées mais complémentaires, Hanan (la moitié masculine haute et supérieure) et Urin (la moitié féminine basse et inférieure), Pachacamac était assimilé à la fertilité, à la fécondité, à la lune, à la nuit, à la Terre et à la mer ; c'est-à-dire au monde « d'en bas ». Pachacamac (Ychsma) était d'ailleurs considéré par les populations de la côte centrale du Pérou comme le créateur du monde et de l'humanité. Il est le dispensateur du souffle de vie. Tandis que son homologue andin, Viracocha, le créateur du monde « d'en haut » et de l'humanité des Incas, était assimilé au Soleil, au jour et au feu solaire. Quant aux mythes, ils présentent de manière récurrente des liens entre Pachacamac, la fertilité, la fécondité et l'abondance (Taylor, 1980 : ch. XXII). II n'est donc pas surprenant qu'il apparaisse ici comme l'époux de la Terre-mère nourricière, la Pachamama, qu'il ressucite et féconde.

À notre sens, la faculté génératrice de la Pachamama, dont il est question dans le mythe, pourrait être ici assimilée aux eaux du fleuve Chillón. En effet, ces dernières naissent du dégel du pic de la Cordillère de la Veuve, soit de l'endroit où elle est enchantée, et irriguent le secteur des basses terres des communautés situées le long du fleuve. Alors, Pachamama serait peut-être pour la vallée haute du Chillón, et même pour son entièreté, une divinité pourvoyeuse d'eau de même que la gardienne des eaux du fleuve. Cependant, contrairement à Villar Córdova, nous ne postulons pas, vu l'insuffisance des données régionales, l'existence d'une telle reconnaissance au niveau régional mais plutôt celle d'une vénération locale.

En ce qui concerne le statut d'apu qu'aurait occupé Pachamama durant les périodes récentes pour la vallée du Chillón, nous remarquons que de manière récurrente des divinités féminines apparaissent, sous des formes diverses (matérielles ou immatérielles issues de la tradition orale), dans la zone étudiée. En effet, les divinités pourvoyeuses d'eau de la vallée haute du 
Chillón sont pour la plupart figurées aux côtés des canaux d'irrigation, des établissements préhispaniques datés de l'Intermédiaire récent, et aujourd'hui abandonnés, sous la forme de huacas (dans ces cas-ci, il s'agit de rochers présentant une forme particulière ou de lieux considérés comme demeures de la divinité) ou de huancas 16 (doubles minéraux des cadavres des ancêtres fondateurs auxquels on rendait un culte comme garants de la fertilité). En nous basant sur les données ethnohistoriques relatives à la vénération de divinités pourvoyeuses d'eau, nous suggérons que les populations de l'Intermédiaire récent de cette vallée, à l'instar des comuneros des communautés voisines actuelles, devaient considérer ces huacas ou huancas comme les maîtresses des eaux, créatrices et gardiennes de leurs canaux et qu'elles réalisaient un nettoyage rituel de ceux-ci en leur rendant hommage.

Actuellement, à Santiago de Huaros, communauté voisine de Huacos, les comuneros vénèrent, lors de la limpiacequia de Jaguajo, une grande pierre travaillée, semblable à une tête humaine, qu'ils appellent la Vieille. Ils la considèrent comme la maîtresse des eaux locales, car elle est située à côté du canal de Jaguajo qui irrigue les basses terres de la communauté. Les comuneros racontent aussi que dans une grotte des hautes terres, située à côté du canal de Mirpo, réside une femme appelée «la Huaca». Ils lui rendent hommage à l'intérieur de la grotte lors de la limpiacequia de Mirpo en tant que déesse du canal car elle pourvoit en eau les hautes terres de la communauté. La communauté de Lachaqui rend hommage lors de sa limpiacequia à la Vieille et celle de Cullhuay, à la Vieille, à la grand-mère et à la Veuve sous forme de huacas situés aux abords ou à l'intérieur de deux lagunes : Riguacocha et Chunchuncocha (Borea, 2004 : 155-156 ; Farfán, 2002 : 132-140 ; Raez, 2001 : 340-343). Et finalement, les comuneros de San Pedro de Huacos vénèrent la Mamahuaca et son huaca, la chute d'eau d'Ongongoy, en sa qualité de divinité pourvoyeuse d'eau et ancêtre créatrice des canaux.

Toutes ces divinités pourvoyeuses d'eau sont féminines et veuves, ce qui est assez exceptionnel car les ouvrages anthropologiques sur le sujet mentionnent pour la plupart des divinités masculines (Rivera, 2002 : 312-317). Cette donnée semble dépendre du contexte historique et culturel de la vallée haute du Chillón. Villar Córdova pense, et nous partageons son avis, qu'à travers les huacas et les huancas des communautés, cités ci-dessus, c'était la Terre-mère nourricière, dans son statut de veuve qui était honorée. Pachamama occupait peut-être jadis, et occupe peut-être encore de manière plus " camouflée », lors des cérémonies propitiatoires, le statut d'apu le plus vénéré par les communautés de la vallée haute du Chillón. Un entretien mené avec un comunero de Huaros confirme cette hypothèse. Il dit à propos de «la Huaca», vénérée lors de la limpiacequia de Mirpo :

« Ils la respectent car c'est la terre, elle fait en sorte que la terre produise, c'est la maman (...) elle fait en sorte qu'à nous tous il ne nous manque pas de nourriture. » (ma trad.) (Santos Hurtado, 2004).

En outre, selon des recherches anthropologiques récemment menées dans la portion haute de la vallée, les joueuses de petit tambourin des limpiacequias doivent obligatoirement être des veuves (Borea, 2004 : 156). Finalement, nous suggérons que d'autres personnages mythiques, tels que le renard (ou la renarde) et la mouffette, présents dans la « danse des parianes » et le mythe des willkas, renforcent l'hypothèse de la vénération de la Terre-mère en tant que veuve du dieu côtier et suggèrent l'existence d'anciennes relations entre les populations du haut Chillón et le secteur côtier du site de Pachacamac.

L'opposition entre les renards et les pumas, qui caractérise la « danse des parianes » de Huacos, est déjà mentionnée dans les sources anciennes. Selon une histoire recueillie,

16 II va de soi que tous les huancas et huacas de la vallée haute ne sont pas de nature féminine ; cependant, il y en a une majorité. 
au XVI ${ }^{e}$ siècle, par un voyageur allemand, le dieu Soleil, Inti, jaloux des sacrifices offerts à Pachacamac, envoya un puma qui tua et mangea le renard, animal favori de ce dieu (Eeckhout, 1999 : 390). Le manuscrit de Huarochirí, raconte que Pariacaca, dieu montagnard, s'éprit de Chuquisuso et pour obtenir ses faveurs, il décida de lui construir une acequia. Pour ce faire, il convoqua plusieurs animaux, dont des renards et des pumas, qui se disputèrent la tâche de tracer son cours. Le renard gagna, mais au milieu de son parcours, il fut effrayé par l'envol d'une perdrix et tomba (Taylor, 1980 : ch. VI). S'il n'était pas tombé, le cours du canal serait situé en amont.

Quant à « la danse des parianes », elle n'est pas sans rappeller la conception dualiste de la société et de l'espace représentée lors de rencontres rituelles de l'Inti Raymi au Cuzco, entre les côtiers et les montagnards. Selon Garcilaso de la Vega (1967 [1609] : ch. XX), le premier jour de cette célébration était marqué par une sorte de défilé des participants. Eeckhout (1999 : 394) suggère que les côtiers y auraient assumé le rôle des renards (des mouffettes, etc.) et les montagnards celui des pumas (des condors, etc.). Selon Zuidema, chez les Incas, le côté Hanan-masculin, c'est-à-dire, le puma montagnard, est associé aux premiers-nés, aux aînés, tandis que le côté Urin-féminin, c'est-à-dire le renard (ou la renarde) côtier, est associé au frère cadet (Zuidema, 1985 : 238. In : Eeckhout, 1999 : 393). Le troisième jour de la limpiacequia d'Ongongoy-Shispo, lors des compétitions sous forme de jeux sur la place centrale de la communauté, c'est généralement le puma qui donnait les ordres et dominait physiquement le renard. Celui-ci, déclaré perdant lui rendait hommage. Dans ce cas-ci, le renard est considéré comme un jeune homme, inférieur au puma qui est considéré comme grand-père ou frère-ainé (Urton, 1985 : 256. In : Eeckhout, 1999 : 393). Mais sur la place principale de Huacos, la domination pouvait s'inverser en faveur de la côte lors du dernier jeu compétitif à la corde. Rappelons que la victoire du groupe du parian déguisé en renard, opposé à celui du parian déguisé en puma, impliquait de bonnes récoltes.

À l'époque incaïque, le renard semble avoir été lié à la période des pluies et au cycle des cultures. Zuidema (1985 : 193. In : Eeckhout, 1999 : 393) précise d'ailleurs qu'à cette époque, c'était l'intensité de ses glapissements qui présageait l'importance des récoltes en mars et en octobre.

Les agriculteurs actuels du Cuzco affirment que ce sont les renards qui, par leurs hurlements, annoncent aux mois d'octobre et de novembre les récoltes de l'année suivante. Si durant cette période, leurs hurlements sont forts, les paysans jouiront, grâce à l'eau de pluie, d'une année prospère; au contraire si les hurlements sont faibles ce sera une année de disette. En Bolivie, les paysans affirment que s'ils voient, au mois de septembre, le renard se diriger vers la zone de la puna, les récoltes y seront bonnes, mais s'il descend dans la vallée c'est elle qui obtiendra de bonnes récoltes. Les excréments des renards sont aussi reconnus, par les cultivateurs, comme des indicateurs agricoles. S'ils contiennent des pelures de pomme de terre, les récoltes seront bonnes dans la puna ; mais si elles présentent des restes de maïs les récoltes seront bonnes dans la vallée. Les agriculteurs actuels du Cuzco et de Bolivie dépeignent le renard comme un animal vorace et stupide (Zuidema, 1989: 319. In : Borea, 2004 : 171-172; Morote Best, 1988).

La pensée de ces comuneros montre une ambivalence certaine envers cet animal ; il est à la fois méprisé car il est considéré comme un voleur d'aliments, mais aussi tenu en vénération car il est considéré comme l'animal annonciateur des pluies à venir. II est frappant de constater que, dans les limpiacequias anciennes et actuelles de la haute vallée du Chillón, on retrouve chez le personnage du parian cette même ambivalence. Jouant le rôle des renards (et des pumas) et parfois appelés comme tels, ils sont à la fois considérés comme pertubateurs, voleurs d'aliments à la mesa et voleurs d'enfants dans la communauté (Borea, 2004 : 19). Mais ils sont aussi les alliés des comuneros car ils les aident durant le nettoyage 
en les encourageant; ils aident également les veuves à récolter les ingrédients pour la préparation du patache. De plus, en faisant les offrandes aux divinités pourvoyeuses d'eau, ils deviennent les intermédiaires entre les comuneros et ces dernières; parfois ils sont même assimilés à ces divinités (Raez, 2001 : 343-349 ; Llanos \& Osterlings, 1981 : 34-36 ; Rivera, $2002: 312-317)$.

Or les sources anciennes (Garcilaso de la Vega, 1967 [1609] : ch. IX ; Cieza de Léon, 1995 [1551] : ch. L), montrent aussi le renard sous un aspect ambivalent. Lorsqu'elles traitent des basses terres et de la côte, elles mentionnent sa vénération au temple de Pachacamac et présentent le renard (ou la renarde) comme un animal astucieux, rusé et courageux. Par contre, lorsqu'elles traitent des hautes terres, elles le présentent comme un animal méprisé pour sa vanité et sa suffisance (Eeckhout, 1999 : 392). Le manuscrit de Huarochirí en montre un bon exemple. Lors de sa course vers la mer, le grand dieu des montagnes Cuniraya Viracocha croisa un renard et lui dit :

«Même si tu te promènes à distance, les hommes te haïront et te traiteront de renard de malheur. Quand ils te tueront, ils te jetteront toi et ta peau comme des choses sans valeur » (Taylor, 1980 : ch. II).

Le renard semble donc avoir été un animal considéré, de manière préférentielle, comme côtier en opposition avec les animaux de la montagne représentés généralement par le condor et/ou le puma. Il en va de même pour la mouffette que Cuniraya maudit également au cours de son périple; en réalité il bénit les animaux qui l'encouragent (le condor, le puma et le faucon) et maudit ceux qui le découragent (la mouffette, le renard et le perroquet).

\section{CONCLUSION}

Il existe vraisemblablement une continuité dans la relation entre le renard et la côte étant donné, qu'à notre sens, les divinités pourvoyeuses d'eau, visibles par une multitude de figurations matérielles, telle que la Vieille, la Veuve, et la Mamahuaca, représentaient de manière symbolique Pachamama, veuve de Pachacamac, dieux côtier dont l'animal favori était le renard. Outre l'association récurrente dans l'aire andine, entre le parian, le renard, la période des pluies et le cycle des cultures, il est possible que les populations de la vallée haute du Chillón, malgré leur situation dans les hautes terres et leurs histoires culturelles particulières, aient été englobées dans l'orbite côtier du site de Pachacamac ou en aient subi l'influence. Ce qui expliquerait la vénération de Pachamama en tant que veuve de Pachacamac et celle du renard ou de la renarde en tant qu'animal allié et pourvoyeur d'eau. Ajoutons que des données archéologiques provenant de sites de la vallée moyenne du Chillón, datés de la période de l'Intermédiaire récent, attestent l'influence côtière sur cette portion de vallée.

Ceci n'est qu'une hypothèse concernant l'origine de la nature des divinités pourvoyeuses d'eau du haut Chillón. De plus amples recherches ethnoarchéologiques et ethnohistoriques dans les communautés de la vallée haute du Chillón seront à même d'éclairer le sujet.

\section{Remerciements}

Je tiens à remercier les professeurs Eeckhout, Malengreau et Petit de l'Université Libre de Bruxelles pour m'avoir aidée de leur conseils avisés. Ma gratitude va également au professeur Farfán, archéologue et professeur de l'Université Nationale Federico Villareal de Lima, qui m'a introduite dans les communautés villageoises de la vallée haute du Chillón et qui m’a prodigué de précieuses recommandations. La recherche de terrain a pu être menée dans de bonnes conditions grâce à 
I'accueil et à la collaboration des habitants de San Pedro de Huacos et de Santiago de Huaros. Je remercie particulièrement Madame Teodolinda Otarola pour la sollicitude dont elle m’a entourée durant mes séjours à Huacos. Lors de mes visites à Huaros, Madame Rosa León, Monsieur Emilio Torres et le Docteur Edwin Santos m'ont communiqué des informations précises et utiles et ont facilité les contacts. Que tous ceux qui m'ont aidée ponctuellement dans mes recherches soient remerciés : Marco López, étudiant en archéologie de I'Université Nationale Federico Villareal de Lima et Victoria Aranguren, archéologue de I'Université Nationale Federico Villareal de Lima et les anthropologues Raez et Borea de l'Université Pontificale Catholique du Pérou.

\section{Références citées}

ARRIAGA, P. J. de, 1999[1621] - La Extirpación de la Idolatría en el Pirú, 200 p. ; Cuzco : Centro de Estudios Rurales Andinos «Bartolomé de las Casas». Estudio preliminar y notas de Henrique Urbano. Monumenta Idolátrica Andina, 3.

BOREA, G., 2004 -Tras los pasos del Parian en el evento y en el tiempo. Imágenes y representaciones en la Sierra de Lima. Anthropológica, 22 : 151-178.

CARRIÓN CACHOT, R., 1955 - El culto al agua en el antiguo Perú. La Paccha elemento cultural Pan-andino. Separata de la Revista Nacional de Antropología y Arqueología, 2 (1) : 1-96.

CASTRO de TRELLES, L., 1992 - Relación de la religión y ritos del Perú hecha por los padres agustinos, 74 p. ; Lima : Pontificia Universidad Católica del Perú. Colección Clásicos peruanos, 8. Première édition.

CHAMORRO, A., 1982 - Sondeo Histórico de un Modelo Islámico en América Hispana. Revista de Música Latinoamericana, 3 (2) : 165-187.

CIEZA DE LEÓN, P., 1995 [1551] - La Crónica del Perú, 354 p. ; Lima : Pontificia Universidad Católica del Perú.

COBO, F. B., 1964 [1653] - Obras del P. Bernabe Cobo II, T. XCII , 515 p. ; Madrid : Ediciones Atlas. Biblioteca de Autores Españoles.

COMUNIDAD CAMPESINA «SAN PEDRO DE HUACOS» : fiche technique (fin des années 1990).

CONDORI, B. \& GOW, R., 1976 - Kay Pacha : tradición oral andina, 99 p. ; Cuzco : Centro de Estudios Rurales Andinos Bartolomé de las Casas.

DILLEHAY, T., 1980 - Relaciones prehispánicas costa-sierra en el valle del Chillón. In : $3^{\mathrm{e}}$ Congreso peruano: El Hombre y la cultura andina: actas y trabajos. Tomo III (Matos Mendieta Ramiro, ed.) : 120-140 ; Lima : Lasontay.

DUVIOLS, P., 1973 - Huari y Llacuaz. Agricultores y pastores. Un dualismo prehispánico de oposición y complementariedad. Revista del Museo Nacional, 39 : 153-191.

DUVIOLS, P., 1977 - La destrucción de las religiones andinas (durante la conquista y la colonia), 479 p. ; México : Universidad Nacional Autónoma de México. Historia General, 9.

EECKHOUT, P., 1999 - Pachacamac durant I'Intermédiaire récent. Étude d'un site monumental préhispanique de la Côte centrale du Pérou, 504 p. ; Oxford : Hadrian Books Ltd. British Archaeological Reports International Series, 747. 
Le nettoyage rituel des canaux d'irrigation d'une communauté de la cordillère de Lima

FARFÁN LOBATÓN, C., 2002 - El simbolismo en torno al agua en la comunidad de HuarosCanta. Bulletin de I'Institut Français d'Études Andines, 31 (1) : 115-142.

GARCILASO DE LA VEGA, I., 1967 [1609] - Comentarios Reales de los Incas, T. I-III ; Lima : Editorial Universo S.A. Colección Autores Peruanos.

GELLES, P., 2002 - Agua y poder en la sierra peruana : la historia y política cultural del riego, rito y desarrollo, 239 p. ; Lima : Pontificia Universidad Católica del Perú.

GOSE, P., 1994 - Deathly waters and hungry mountains: agrarian ritual and class formation in a Andean town, 325 p. ; Toronto : University of Toronto Press. Anthropological horizons, 4.

GUAMAN POMA DE AYALA, F., 1987 [1615] - El primera nueva crónica y buen gobierno, T. I-III ; Madrid. Murra, J., R. Adorno et J. Urioste, eds. Crónicas de America, 29a-c, Historia, 16.

ISBELL, B. J., 1978 - To defend ourselves: ecology and ritual in an andean village, 289 p. ; Austin : University of Texas Press. Latin American monographs series.

LECAROS, A. T., 1996 - La fiesta del agua de Mama Capiama. Ritos y tradiciones en torno a un personaje mítico, 132 p. ; Lima : Pontificia Universidad Católica del Perú. Facultad de Ciencias Sociales, Tesis de Licenciatura.

LLANOS, O. \& OSTERLINGS, J., 1981 - Ritual de la fiesta del agua en San Pedro de Casta ; Lima : Pontificia Universidad Católica del Perú.

MALENGREAU, J., 1995 - Sociétés des Andes. Des empires aux voisinages, 454 p. ; Paris : Éditions Karthala. Collection Hommes et sociétés.

MARISCOTTI de GÖRLITZ, A. M., 1973 - La posición del señor de los fenómenos meteorológicos en los panteones regionales de los Andes Centrales. Revista del Museo Nacional de Historia, 6 : 207-15.

MÉTRAUX, A., 1983 - Les Incas, 190 p. ; Paris : Éditions du Seuil. Collection Points Histoire.

MOROTE BEST, E., 1988 - El tema del viaje al cielo. In : Aldeas sumergidas: Cultura popular y sociedad en los Andes (Efraín Morote Best ed.) : 55-100 ; Cuzco : Centro de estudios rurales andinos Bartolomé de las Casas.

ORTIZ, A., 1973 - De Adaneva a Inkarri, 191 p. ; Lima : Ediciones Retablo de Papel.

OSSIO, J., 1992 - Parentesco, reciprocidad y jerarquía en los Andes. Una aproximación a la organización social de la communidad de Andamarca, 407 p. ; Lima : Pontificia Universidad Católica del Perú-Fondo Editorial.

RAEZ, M., 2001 - Jerarquía y autoridad comunal. Los varayos y la fiesta del agua de la comunidad campesina de Lachaqui, Canta. In : Identidades representadas. Performance, experiencia y memoria en los Andes (Gisela Cañepa Koch, ed.) : 332364 ; Lima : Pontifica Universidad Católica del Perú-Fondo editorial.

RIVERA, J., 2002 - Ritos agrícolas en el valle del Chancay (Lima). Testimonios sobre las celebraciones en torno a la limpieza de acequia recopilados cuarenta años después. Anthropológica, (20) : 309-332.

ROSTWOROWSKI de DIEZ CANSECO, M., 1978 - Señoríos Indígenas de Lima y Canta, 280 p. ; Lima : Instituto de Estudios Peruanos.

ROSTWOROWSKI de DIEZ CANSECO, M., 1988 - Conflicts over coca fields in XVI th Century Peru, 314 p. ; Michigan : University of Michigan Museum of Anthropology. Memoirs of the Museum of anthropology university of Michigan, vol. 21, Studies in Latin American Ethnohistory and Archaeology, 4.

SALLNOW, M. J., 1987 - Pilgrims in the Andes. Regional Cults in Cusco, 351 p. ; Washington D.C. : Smithsonian Institution Press. 
SALOMON, F., 1997 - Collquiri's Dam : The Colonial Re-Voicing of an Appeal to the Archaic. In : Native Traditions in the Postconquest World (Elizabeth Hill \& Cummins, T., eds) : 265-293 ; Washington D.C. : Dumbarton Oaks Research Libraries and Collections.

SANTOS HURTADO, E., 2004 - Comunidad campesina de Huaros [document de travail].

SHERBONDY, J., 1979 - Les réseaux d'irrigation dans la géographie politique du Cuzco. Journal de la Société des Américanistes de Paris, 66 : 45-66.

SHERBONDY, J., 1987 - Organización hidráulica y poder en el Cuzco de los Incas. Revista española de antropología Americana, 17 : 117-153.

SILVA, J. E., 1996 - Prehistoric settlement patterns in the Chillón river valley, Peru, 628 p. ; Michigan : Ann Arbor, University of Michigan.

TAYLOR, G., 1980 - Rites et Traditions de Huarochirí : manuscrit quechua du début du 17e siècle, 243 p. ; Paris : Éditions L'Harmattan.

TELLO, J. C. \& MIRANDA, P., 1923 - Wallallo, Capitulo 5 : Las ceremonias gentilicas en honor de Wallallo. Inca, 1 (3) : 523-545.

URTON, G., 1981 - At the Crossroads of the Earth and the Sky : an Andean cosmology, 248 p. ; Austin : University of Texas.

VALCÁRCEL, M. \& LUMBRERAS, K., 1997 - Agricultores de Chillón: modernización e institucionalidad, 128 p. ; Lima : Fomento de la vida.

VALDERRAMA, R. \& ESCALANTE, C., 1988 - Del Tata Mallky a la Mamapacha. Riego, Sociedad y Ritos en los Andes Peruanos, 243 p. ; Lima : Centro de Estudios y Promoción del desarrollo.

VARGAS, H., 1990 - La comunidad campesina de Laraos y el rito de la champería. Anthropológica, 8 : 195-214.

VILLAR CÓRDOVA, P. E., 1923 - Las ruinas de la provincia de Canta. Inca 1 (1) : 1-15.

VILLAR CÓRDOVA, P. E., 1933 - Folklore de la Provincia de Canta (En el Departamento de Lima). El mito «Wa-kon y los Willkas» referente al culto indígena de la Cordillera de «La Viuda». Revista del Museo Nacional, 2 (2) : 161-179. 